\title{
Looking for the Optimal Cryptocurrency Portfolio: Benefits of Diversification
}

\begin{abstract}
Tian Chen ${ }^{1, *}$
${ }^{1}$ Electrical Engineering, Elect, Comp \& Syst Enginr

*Jared.chentian@hotmail.com

ABSTRACT

This paper contributes to the literature on cryptocurrencies by examining performance of naïve $(1 / \mathrm{N})$ and individual cryptocurrency portfolio of ten largest cryptocurrencies. We employ daily data and two time durations for each type of portfolio. For the investing in individual cryptocurrency portfolio, the first time duration is the full time duration from the earliest day in our data sample. The second time duration is after Covid-19. (after 1st of January 2020). For the naïve portfolio, the first time duration is the same as individual cryptocurrency portfolio. However, the second time duration is when all ten cryptocurrencies are available. (after November 10th 2018) Our result shows that investing in single cryptocurrency portfolio outperforms than naïve portfolio in both time duration. Further research could add the better performing cryptocurrency portfolio to the traditional assets portfolio and examine the performance of the combining portfolio in different time duration.
\end{abstract}

Keywords: Cryptocurrencies, Nä̈ve diversification, Individual cryptocurrency portfolio, Sharpe raito

\section{INTRODUCTION}

The goal of this paper is to figure out whether investing in a number of cryptocurrencies is better than investing in individual cryptocurrency. It is particularly important because we are now in the economic recession. Stock market in the U.S has fused several times in recent months. In the past, people used to believe that during panic time, gold is the only asset which did not lose value. However, the gold price has decreased during the past economic panic. Although it has recovered since and one month since the crisis begun and it is trading higher than before, the fluctuation of the gold price indicates that investors cannot rely only on gold to maintain their wealth during the economic recession. Therefore, in this essay we propose other assets - cryptocurrencies - which can help investors survive economic panic. The first cryptocurrency Bitcoin was created after 2008, the Great Financial Crisis. Bitcoin was designed for a global crisis. Bitcoin was designed to work on a standalone basis because it doesn't need banks, governments, international currencies or whatever system is needed for traditional fiat money. Only satellite and mesh network communication systems are currently in use in order to keep the Bitcoin blockchain alive even during an internet outage. The low correlation between Bitcoin and the traditional stock market is another factor that might aid a new wave of inflow as investors noticed that crisis has impacted almost all kinds of asset. In consequence, cryptocurrencies tend to be an indispensable asset in order to limit the portfolio risk especially during economic panic time. Hence, deciding how to invest in the cryptocurrency market becomes a major task. The essay asserts that investing in a single cryptocurrency is better than investing in a number of cryptocurrencies.

In order to test the hypothesis stated above, the essay first analyses various cryptocurrencies because we need individual cryptocurrency data to construct relevant portfolio. Obviously, correlations between cryptocurrencies matters. The essay creates two cryptocurrency portfolios. The first one invests in individual cryptocurrencies and the other one in the naïve cryptocurrency portfolio. Firstly, the essay analyses correlations between various cryptocurrencies during two time periods: full duration of the sample (2013.04.29 to 2020.05.06) and the duration after COVID-19 outbreak (1st of January 2020). With knowledge of the correlation between various cryptocurrencies, we construct two cryptocurrency portfolios and analyse the average return, standard deviation and Sharpe ratio for each portfolio. At last, we compare two portfolios base on the data we analysed and make a conclusion about which portfolio behaves better. 
The plan of the essay is as follows. First, we analyse the related literature on cryptocurrencies and their performance during economic crisis. Second, we describe the data; in particular we discuss the data source, data time duration and issues with the data. Thirdly, we analyse the data step by step with the goal of finding the best cryptocurrency portfolio. Finally, we conclude.

\section{RELATED LITERATURE}

We first review the impact of various diversification methods by considering their impact on performance. There is very little difference in terms of expected returns, Sharpe ratio and Omega ratio between naïve diversification and optimal diversification indicating that the gain from optimal diversification is more than offset by estimation error, consistent with the findings of DeMiguel for equities [1]. Brauneis and Mestel (2018) identify naively diversified portfolios to derive riskadjusted outperformance when compared to meanvariance optimized portfolios [2]. They also find that combining cryptocurrencies enriches the set of 'low'-risk cryptocurrency investment opportunities. Hence, the 1/N -portfolio outperforms single cryptocurrencies and more than $75 \%$ of mean-variance optimal portfolios in terms of the Sharpe ratio and certainty equivalent returns.

There is ongoing debate about the reasons why investors hold cryptocurrencies. Cryptocurrencies have been shown to be held by investors as speculative assets rather than a currency [3]. Bitcoin market fundamentals and Bitcoin's attractiveness for investors have a significant impact on Bitcoin price [4]. However, there is empirical evidence that bubble behaviour of Bitcoin and Ethereum is repeated [5]. Bitcoin is more an investment than a currency because Corbet's results show that there is a huge difference between the behaviour of Bitcoin returns from conventional currency returns when comparing both aspects of level and stability. When the speculation is exaggerated to an unrealistic level that results in overvaluation of the assets beyond the true value that reflects the intrinsic value of the asset, the bubble behaviour of Bitcoin starts.

Finally, we review the existing papers which examine the impact of the correlation between cryptocurrencies in different situation. Liu (2018) finds that diversification among the cryptocurrencies can significantly enhance the Sharpe ratio and utility [6]. He also concludes that naïve portfolio outperforms in terms of Sharpe ratio criterion because the result shows that correlation between cryptocurrencies in a naïve portfolio is relatively low. However, there are some evidences that correlation between cryptocurrencies is extreme during some time period. In particular, Gkillas, Bekiros and Siriopoulos (2018) find that extreme correlation is not related to cryptocurrency market volatility per se, but to the trend of the cryptocurrency market which means extreme correlation increases in bear markets, but not in bull market for cryptocurrency pairs [7]. However, there is also a non-negligible number of pairs which exhibit a weak level of dependency in extremes. On the other hand, different cryptocurrency markets could be interdependent both in the short run and long run. Ciaian et al. (2018) study a significantly large number of cryptocurrencies and conclude that Bitcoin and altcoin markets are interdependent both in the short run and the long run [8]. Nevertheless, some cryptocurrencies' dynamics are relatively isolated to the others which may offer diversification benefits for investors [9].

\section{DATA}

Our data came from www.coinmarketcap.com aggregator. We selected 10 large cryptocurrencies whose market capitalisation is over 1 billion dollars: Bitcoin (2013.04.29-2020.05.06), Litecoin (2013.04.292020.05.06), Bitcoin SV (2018.11.09-2020.05.06), Ethereum (2015.08.08-2020.05.06), XRP (2013.08.052020.05.06), Bitcoin Cash (BCH) (2017.07.242020.05.06), Binance Coin (BNB) (2017.07.252020.05.06), EOS (2017.07.02-2020.05.06), Tezos (XTZ) (2018.06.30-2020.05.06) and Stellar (XLM) (2014.08.05-2020.05.06). The data includes the close price of each cryptocurrency. All the data explanations are in the table 1.

Table 1. Data explanation

\begin{tabular}{|c|c|c|}
\hline $\begin{array}{c}\text { Cryptocurrency } \\
\text { Name }\end{array}$ & Start Time & End Time \\
\hline Bitcoin & April 29th 2013 & May $6^{\text {th }} 2020$ \\
\hline Litecoin & April 29th 2013 & May 6rh 2020 \\
\hline Bitcoin SV & $\begin{array}{c}\text { November } 9^{\text {th }} \\
2018\end{array}$ & May 6rh 2020 \\
\hline Ethereum & August 8 2015 & May $6^{\text {th }} 2020$ \\
\hline XRP & August $5^{\text {th }} 2013$ & May $6^{\text {th }} 2020$ \\
\hline Bitcoin Cash & July 24th 2017 & May $6^{\text {th }} 2020$ \\
\hline Binance Coin & July 25rh 2017 & May $6^{\text {th }} 2020$ \\
\hline EOS & July $2^{\text {nd }} 2017$ & May $6^{\text {th }} 2020$ \\
\hline Tezos & June $30^{\text {th }} 2018$ & May $6^{\text {th }} 2020$ \\
\hline Stellar & August $5^{\text {th }} 2014$ & May $6^{\text {th }} 2020$ \\
\hline
\end{tabular}

There are some issues associated with the data that we need to address. First of all, for some cryptocurrencies like BSV, there is limited data available (less than 2 years). It is not the unavailability of data but the fact that some coins did not exist previously, so the data could not be obtained. When we comprise two different Cryptocurrencies into single portfolio, the time duration of the portfolio should be the time duration of the cryptocurrency which has a shorter history. This may influence the behaviour of the cryptocurrency which has a longer history because some of its data are not included 
in the portfolio. Secondly, there is no one uniform price because cryptocurrencies are traded on several exchanges. Thus, there may be some cryptocurrency prices might be incorrect. This inconsistency will however only slightly influence the analysis of the data. Lastly, the 10 largest cryptocurrencies by market capitalisation were selected on May 14th 2020, but the ranking is changing and thus the selection may fluctuate. A more robust method of top-10 cryptocurrency selection may be needed.

\section{ANALYSIS}

As explained in the introduction, we analyse how an optimal portfolio consisting of top-ten cryptocurrencies can be constructed.

For the first step, I begin by considering the correlations between the cryptocurrencies in my sample. Correlations are calculated by taking the following steps. First, we find the mean of all the $\mathrm{X}$ values. Second, we find the standard deviation of all the $X$ values and the standard deviation of $Y$ values. Third, for each of the $n$ pairs $(X, Y)$ in the data set, we take $\mathrm{x}$ minus the mean of $\mathrm{X}$ and $\mathrm{y}$ minus the mean of $\mathrm{y}$ and then multiply them together to get (X-mean of X) (Y- mean of Y). Fourth, we add up the $\mathrm{n}$ results from step 3 . Fifth, we divide the sum by (standard deviation of $\mathrm{X}$ multiply the standard deviation of $Y$ ). Finally, we divide the result by $n-1$, where $\mathrm{n}$ is the number of $(\mathrm{X}, \mathrm{Y})$ pair. The correlation formula is down below.

$$
r=\frac{1}{n-1}\left(\frac{\sum_{\mathrm{x}} \sum_{\mathrm{y}}(\mathrm{x}-\overline{\mathrm{x}})(\mathrm{y}-\overline{\mathrm{y}})}{\mathrm{s}_{\mathrm{x}} \mathrm{s}_{\mathrm{y}}}\right)
$$

Jupyter notebook is the tool to analyse the data. First, I consider the full time period of the sample. This is supposed to give us an estimate for long-term behaviour of the cryptocurrencies; for most coins the time period involves various types of market regime such as bull run, bust and sideways market. This duration is determined for each pair of cryptocurrencies by the currency with a shorter history. For example, for the correlation coefficient between EOS and Binance Coin, the time period covered is 25th of July 2017. The result is illustrated in Table 2 below.

Table 2. The correlations between cryptocurrencies for the full duration of the sample

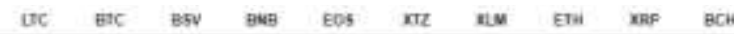

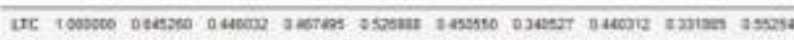

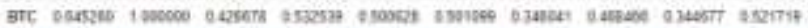

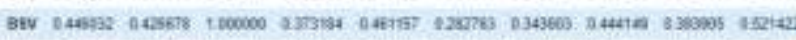

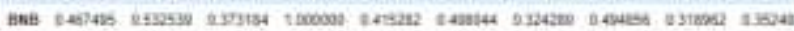

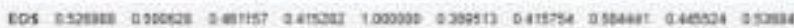

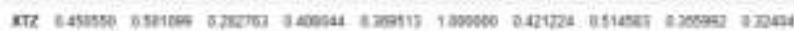

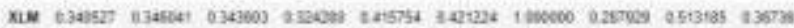

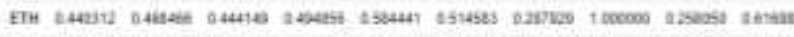

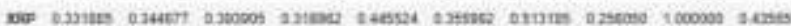

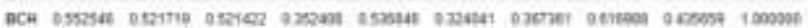

The results run against the popular belief that all cryptocurrencies are highly correlated. In fact, the highest observed correlation is 0.65 between Bitcoin and Litecoin. The average correlation is only 0.43 with the median at 0.44 and the minimum at 0.26 . In fact, the correlations for each individual cryptocurrency are relatively low, ranging between 0.37 and 0.48 ; it is not the case that we have a mixture of high-correlation and extremely low-correlation currencies. Bitcoin shows the highest correlations, which is not surprising because it is by far the largest cryptocurrency and thus sees as a leader that is followed by other coins. These relatively low correlations seem to suggest that there might be significant benefits from diversification to be obtained when constructing the crypto-currency portfolio.

The other time period we consider is following the COVID-19 outbreak: 1st of January 2020. The reason of choosing this specific period is because it was the most recent time when stock market fused and the behaviour of the cryptocurrencies could be representative. The result is illustrated in Table 3 below.

Table 3. The correlations between cryptocurrencies for the COVID-19 outbreak duration of the sample

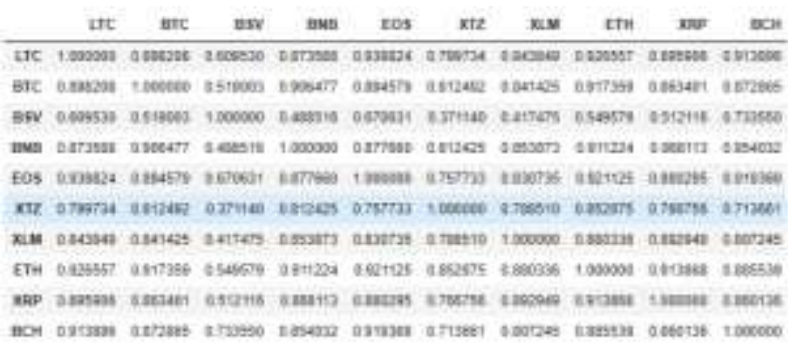

The results in the second period are different than those in the full duration sample. The correlation following the COVID-19 outbreak is much higher than the full duration sample for several reasons. Firstly, the time span limits to only few months which only includes a few amounts of available data. Few amounts of data could result in extreme high correlation between cryptocurrencies. Secondly, the whole financial market was bear market after COVID-19 outbreaks which could cause the large drawdown in the whole cryptocurrency market. However, the second reason will hold true only for the period immediately following the outbreak (March \& April), otherwise the markets rebounded strongly. The largest correlation is between EOS and LTC: 0.9398, and the smallest correlation is between XTZ and BSV: 0.3711 . The average correlation is 0.80 with the median at 0.85 and the maximum at 0.94 . Due to strongly high correlation after the COVID-19 outbreak, it seems to be the case that the diversification in a cryptocurrency portfolio might not be efficient during the bear market.

Next, I consider the performance of two types of portfolios, investing in individual coins and in an equallyweighted portfolio, for each of the two time periods discussed above. This is supposed to give us an answer as to which portfolio would be a better cryptocurrency 
portfolio. The first time period is the full duration sample and the second period is from 2018.11.10 to the end when all coins are available. The second time period is different from the second period defined in the earlier analysis. The earlier second time period is after COVID-19 outbreak. However, the second period here is from the date when all coins are available to today.

The first portfolio invests in individual coins. Average return, standard deviation and Sharpe ratio are the parameters to evaluate the portfolio and are listed in the Table 4 below.

Table 4. Individual cryptocurrencies' portfolio performance for the full duration of the sample

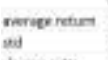

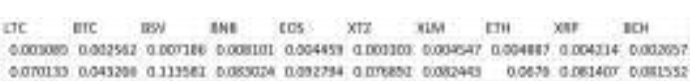

Base on the table above, BNB has the highest Sharpe ratio 0.0976 which means it is the best assets to invest among other cryptocurrencies. Beyond that, BSV has the highest standard deviation 0.1136 which means it has the highest investing risk.

The second period is from 2018.11.10 when all coins become available. Results are shown below:
Table 5. Individual cryptocurrencies' portfolio performance for the second period of the sample

serd

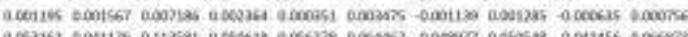

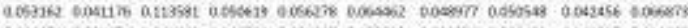

Base on the table, BSV has the highest Sharpe ratio 0.0632 which means it is the least bad asset to invest compare to all other cryptocurrencies. However, BSV also has the highest standard deviation 0.1136 which means investing risk is high. BSV has the highest standard deviation during both periods. Comparing the Sharpe ratio during the second period to the full duration of the sample, Sharpe ratio of the full duration sample is significantly greater than the second period. This probably results from the cryptocurrency bubble behaviour. Bubble behaviour of cryptocurrency will have a more serious impact on the shorter period portfolio. In consequence, the standard deviation of the second period portfolio will larger than the full duration sample portfolio. Beyond that, some cryptocurrencies have negative average return, like XRP, XLM, indicating that cryptocurrency market is not stable in recent years.

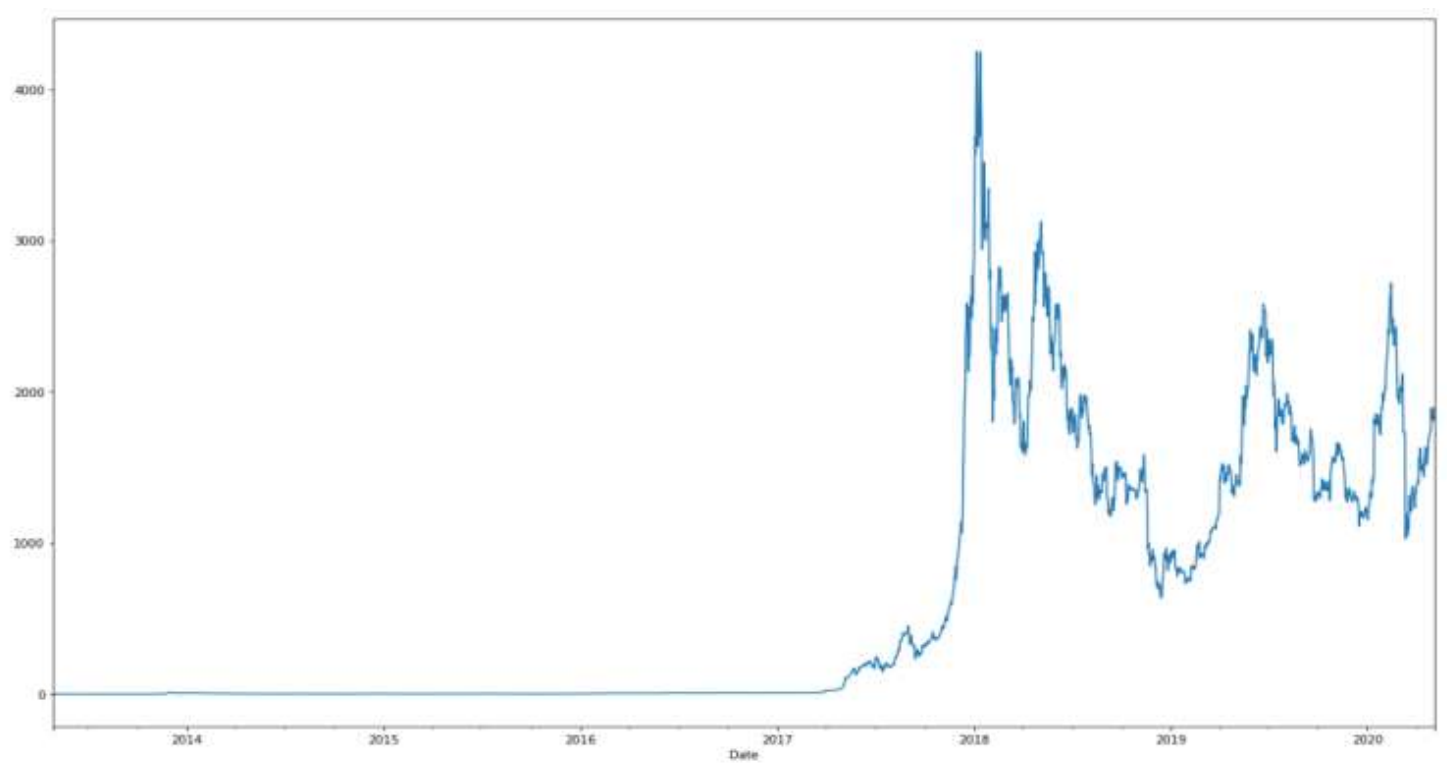

Figure 1. Performance of the naïve portfolio for the full duration of the sample

As for the second type of portfolio, the equallyweighted portfolio, below is the plot of the naive portfolio for the full duration of the sample. The first time period is the full duration sample and the second period is from 2018.11.10 when all coins are available. The full duration sample indicates that we include the existing cryptocurrencies into the

portfolio at each day. However, the second period (after November 10th 2018) represents all ten cryptocurrencies are in the portfolio at each day. It's two separate model I applied. According to Figure 1 below, X axis is the time and $\mathrm{Y}$ axis is the dollar. The Sharpe ratio is 0.081 , with the average return of $0.43 \%$ and the standard deviation of $5.29 \%$. Comparing to the first type of portfolio for the full duration of sample, the Sharpe ratio is slightly lower than the highest Sharpe ratio of investing in individual coin during the same time duration. However, there is a problem associating with selecting the best-performing coin before its performance 
is actually realised. Because the best-performing coin I chose is based on historical data in the past, the coin I chose may not be the best-performing coin in the future. For the single cryptocurrency portfolio, best-performing coin first should be decided before its performance is actually realised. Hence, I can compare with the average Sharpe ratio for the investments in individual cryptocurrencies.

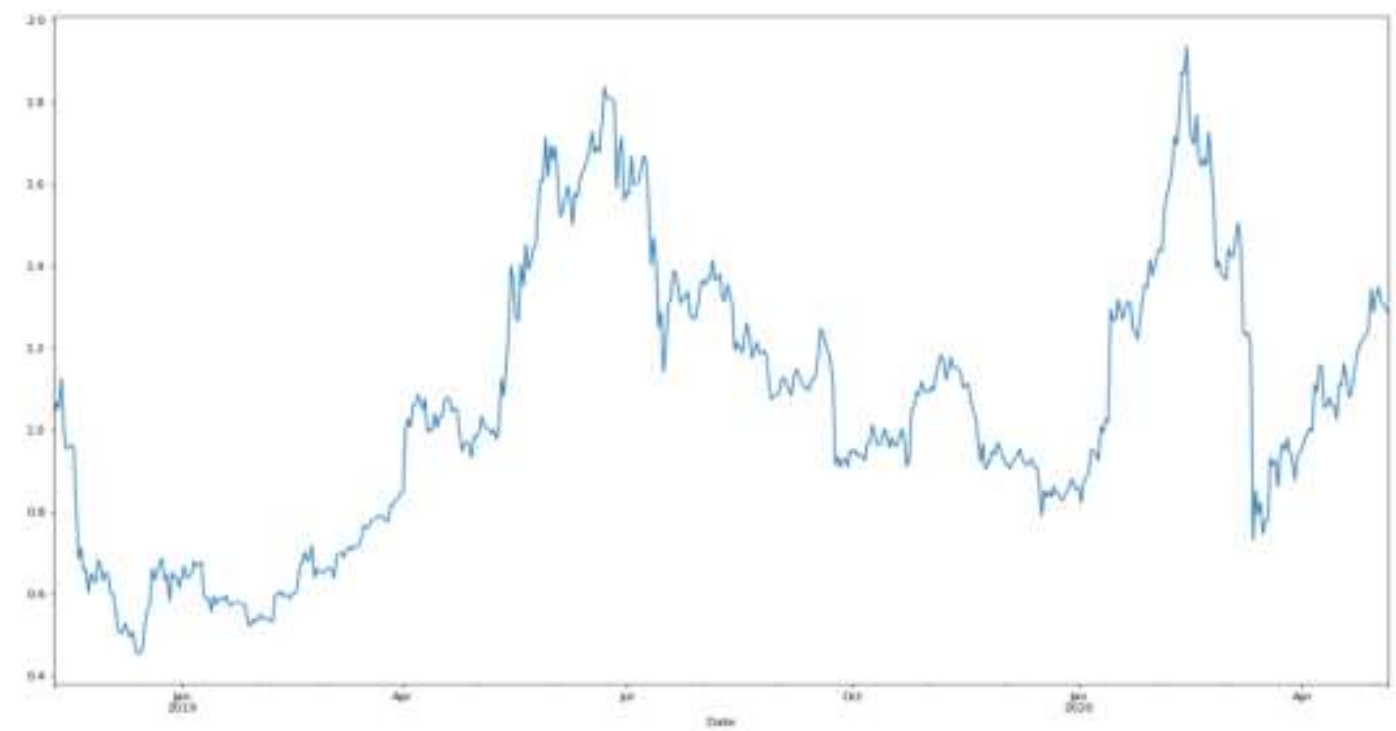

Figure 2. performance of the naïve portfolio for the second period of the sample

For the second period, when all coins are available, below is the plot of the naive portfolio for the second period. According to Figure 2, $\mathrm{X}$ axis is the time and $\mathrm{Y}$ axis is the dollar. The Sharpe ratio is 0.034 , with the average return of $0.16 \%$ and the standard deviation of $4.77 \%$. Comparing to the first portfolio for the second period of sample, the Sharpe ratio is way lower than the highest Sharpe ratio of investing in individual coin during the same time duration.

Since the first portfolio which is investing in individual coins outperforms in both periods comparing to the second portfolio which is the naïve portfolio, the cryptocurrency portfolio should be investing in individual coins.

Since both portfolios rebalance period is daily, the rebalancing cost should be considerable. If the rebalancing cost is 0.001 , the Sharpe ratio of the naïve portfolio for the whole dataset period is decreasing to 0.062 . Therefore, rebalancing cost is a crucial part of the analysis process. It will influence the result to a significant degree.

\section{CONCLUSION}

The correlations between cryptocurrencies over a longer time period are lower than conventionally believed. However, in times of cryptocurrency market stress such as during the COVID-19 outbreak, correlations between cryptocurrencies rise sharply. Therefore, this happens to the extent that it is difficult to obtain any sensible level of diversification which means naïve portfolio will not reduce the risk too much because of the high correlation between cryptocurrencies during the market stress period. Overall, investing in any cryptocurrency over the examined periods would lead to poor Sharpe ratios, with low returns and very high volatility. Being able to identify the winner cryptocurrency would obviously have a positive impact on the performance, but as this is not feasible, investing in an equally-divided (naïve) portfolio might be a better choice.

There are several weaknesses of our paper. First, rebalancing cost could change the research results by a certain degree. Second, the ten biggest cryptocurrencies whose capitalization market value are over 1 billion changed as time goes by. The fluctuating ranking of the cryptocurrencies may result in different results. Third, there is no one uniform price because cryptocurrencies are traded among different

exchanges. Lastly, the way I chose to make up the cryptocurrency's portfolio may not represent the most effective cryptocurrencies portfolio. There are some other ways to form cryptocurrencies portfolio which probably could generate higher Sharpe ratio.

Apart from the weaknesses of our paper, there are some possible extensions of our research. Firstly, once the best performing cryptocurrency portfolio is selected, we can add the cryptocurrency portfolio into the traditional asset portfolio in order to help investors reduce the risk especially during economic panic time such as recent event, COVID-19. Secondly, the research can go deeper to dig the relations between individual cryptocurrencies and the reason why they have the specific connection which will strength the understanding 
of cryptocurrency market. Finally, research could be more extensive by adding more methods of forming cryptocurrencies' portfolio to compare. This will result in a more persuasive and precise conclusion.

\section{REFERENCES}

[1] E. Platanakis, C. Sutcliffe and A. Urquhart, Optimal vs naïve diversification in cryptocurrencies, in: Economics Letters, vol. 171, 2018, pp. 93-96. DOI: $10.2139 /$ ssrn. 3124832

[2] D. Baur, K. Hong and A. Lee, Bitcoin: Medium of exchange or speculative assets?, in: Journal of International Financial Markets, Institutions and Money, vol. 54, 2018, pp. 177-189.

[3] P. Ciaian, M. Rajcaniova and d. Kancs, The economics of BitCoin price formation, in: Applied Economics, vol. 48, no. 19, 2015, pp. 1799-1815.

[4] S. Corbet, B. Lucey and L. Yarovaya, Datestamping the Bitcoin and Ethereum Bubbles, in: Finance Research Letters, vol. 26, 2018, pp. 82-88.
[5] W. Liu, Portfolio diversification across cryptocurrencies, Finance Research Letters, vol. 29, 2019, pp. 200-205.

[6] K. Gkillas, S. Bekiros and C. Siriopoulos, Extreme Correlation in Cryptocurrency Markets, in: SSRN Electronic Journal, $2018 . \quad$ DOI: 10.2139/ssrn.3180934

[7] P. Ciaian, M. Rajcaniova and d. Kancs, Virtual relationships: Short- and long-run evidence from BitCoin and altcoin markets, in: Journal of International Financial Markets, Institutions and Money, vol. 52, 2018, pp. 173-195. DOI: 10.1016/j.intfin.2017.11.001.

[8] S. Corbet, A. Meegan, C. Larkin, B. Lucey and L. Yarovaya, Exploring the dynamic relationships between cryptocurrencies and other financial assets, in: Economics Letters, vol. 165, 2018, pp. 28-34. DOI: 10.1016/j.econlet.2018.01.004 [ 